\title{
Reality/Fiction Distinction and Fiction/Fiction Distinction during Sentence Comprehension
}

\author{
Jie Yang ${ }^{1, *}$, Jin $X^{2} e^{2}$ \\ ${ }^{1}$ Department of Cognitive Science, Macquarie University, Australia \\ ${ }^{2}$ School of English Language, Literature and Culture and Center for Language and Cognition, Beijing International Studies University, \\ China
}

Copyright (C) 2015 by authors, all rights reserved. Authors agree that this article remains permanently open access under the terms of the Creative Commons Attribution License 4.0 International License

\begin{abstract}
The current study examined the cognitive mechanisms of distinguishing reality from fiction and distinguishing different fictional worlds. In Experiment 1, participants read sentences containing reality/fiction mismatch or fiction/fiction mismatch. Results showed that reality/fiction mismatch elicited a N400 effect and a late positivity relative to correct sentences, whereas fiction/fiction mismatch induced a N400 effect. Experiment 2 investigated the reality/fiction distinction after controlling personal relevance. Participants read sentences containing famous people or fictional characters with low personal relevance. Results indicated that reality/fiction mismatch elicited a late positivity in the middle and posterior sites, whereas the pure low relevance showed a late positivity in the posterior sites. The mechanisms under reality/fiction distinction, fiction/fiction distinction, and high/low relevance distinction are discussed.
\end{abstract}

Keywords Fiction, Reality, Personal Relevance, Event-related Potential, Sentence Comprehension

\section{Introduction}

Although living in the real world, people create various fictional worlds through imagination (Lewis, 1978). These fictional worlds can be modern or ancient, the characters can be humans or animals, and events in these worlds can be realistic (e.g., a lumberjack was cutting trees) or unrealistic (e.g., a sausage was singing). It is surprising that people can easily distinguish these fictional worlds from the real world, and one fictional world from another.

Recent studies have investigated how the brain processes reality and fiction during language comprehension. Using fMRI technique, Abraham, von Cramon, \& Schubotz (2008) found that compared with comprehending sentences containing fictional characters (e.g., Peter heard about Cinderella on the radio), comprehending sentences containing real people (e.g., Peter heard about George Bush on the radio) showed stronger activation in the anterior medial prefrontal cortex (amPFC, BA 10) and precuneus / posterior cingulate cortex (PCC) (BA 23/31). These effects imply that processing realistic events involves brain regions related to self-referential processing and episodic memory retrieval (Cavanna \& Trimble, 2006; Gilbert, Dumontheil, Simons, Frith, \& Burgess, 2007; Ramnani \& Owen, 2004). Abraham and Yves von Cramon (2009) further found that personal relevance could be the critical factor underlying reality/fiction distinction: comprehending events involving family or friends (high relevance), famous people (medium relevance), and fictional characters (low relevance) elicited a graded pattern of activity in the amPFC, precuneus and PCC.

Using event-related potentials (ERPs), researchers have examined the time course of comprehending sentences describing fictional events (Ferguson, Sanford, \& Leuthold, 2008; Filik \& Leuthold, 2008; Hald, Steenbeek-Planting, \& Hagoort, 2007; Nieuwland, 2013; Nieuwland \& Martin, 2012; Nieuwland \& Van Berkum, 2006). These studies have shown that fictional linguistic context (e.g., scientific fiction or cartoons) could prevail over local semantic anomalous caused by reality violation (e.g., Filik \& Leuthold, 2008; Nieuwland \& Van Berkum, 2006). Researchers also have found that during sentence comprehension, counterfactual context (e.g., If N.A.S.A. had not developed its Apollo Project...) could eliminate interpretive problems caused by local reality violation (e.g., the first country to land on the moon would have been Russia surely) (Nieuwland, 2013; Nieuwland \& Martin, 2012). Overall, these findings have shown that fictional context can reduce N400 effect elicited by local reality violation. The N400 is assumed to reflect the integration of incoming utterances into preexisting context, and the effect becomes larger when the incoming information is unexpected or anomalous (Kutas \& Federmeier, 2011). The finding that local reality violation can be overridden by fictional context suggests that during sentence comprehension, the reality of events described by incoming information and preexisting context are integrated very fast. 
So far few studies have investigated the distinction different fictional worlds. Evidence from development research indicates that children can distinguish characters from different fictional worlds (Skolnick \& Bloom, 2006). The authors found that children about 4 years old reported that characters from different worlds are fictional to each other. This response pattern is similar to that in the adult group and suggests that fiction/fiction distinction develops very early.

Yang and Xue (2014) using ERPs studied the distinction between realistic fictional events (e.g., Premier Wen Jiabao was talking with President G.W. Bush, and suddenly Colin Powell came into the room) and unrealistic fictional events (e.g., Harry Potter was talking with Hermione, and suddenly Lord Voldemort appeared). By exchanging the character's name in the second clause of the two types of sentences, the authors generated realistic-inconsistent and unrealistic-inconsistent conditions. The ERP result showed that the inconsistency of the reality/fiction elicited a larger late positivity as compared with consistent sentences. According to the situation model theories (e.g., Zwaan \& Radvansky, 1998), comprehenders build a mental representation of the described situation during language comprehension. The situation model is updated whenever incoming language information describes a change of the important dimensions in a situation, such as a new character, a new object, new time or spaces, or a character's new intention. The larger late positivity in the inconsistent sentences indicated that information about reality or fiction can constrain situation model updating, and that reality/fiction mismatch between the incoming information and preexisting context can cause difficulty at a late stage in comprehension.

Although this finding provides evidence for the distinction of realistic and unrealistic fictional events, it is still unknown how the brain distinguishes different unrealistic fictional worlds (e.g., distinguishing the world of spider-man from the world of Harry Potter). Furthermore, it is unclear whether reality/fiction distinction is related to other factors, such as personal relevance. Abraham and Yves von Cramon (2009) showed that the different effects elicited by famous people's names and fictional characters' names may reflect different levels of personal relevance in the two types of entities. Famous people's names may have higher personal relevance to comprehenders as compared with fictional characters' names. Thus, the distinction between reality and fiction might reflect the distinction between high and low personal relevance.

The current study aims to address the above issues. In the first experiment, we used ERPs to examine the brain effects associated with reality/fiction distinction and fiction/fiction distinction. Participants read sentences and their brain responses were recorded. The sentences described fictional events and all characters may be from the same fictional world, or the sentences described fictional events but they may contain famous people in the real world or fictional characters from another fictional world. Given the fact that acceptability judgment task may enhance the late positivity while eliminating the N400 effect (Yang \& Xue, 2014), a semantic plausibility judgment task was used in the current study. The hypothesis is that if reality/fiction distinction and fiction/fiction distinction play the same role during situation model updating, the two types of anomalies would induce similar processing difficulties reflected by the N400 effect (Ferguson et al., 2008; Filik \& Leuthold, 2008; Hald et al., 2007; Nieuwland, 2013; Nieuwland \& Martin, 2012; Nieuwland \& Van Berkum, 2006) or late positivity (Yang \& Xue, 2014). If the two types of anomalies elicit different processing difficulties, then different ERP effects would be observed.

In the second experiment, we examined the brain response to the reality/fiction distinction while controlling personal relevance. The presented sentences described familiar daily events but may contain famous people or fictional characters that have very low personal relevance to the participants. The hypothesis is that if reality/fiction distinction merely reflects the distinction between high and low personal relevance, then the two types of anomalies would elicit similar ERP effects. On the other hand, if the reality/fiction distinction is different from relevance processing, then the two anomalies would show different ERP effects.

\section{Experiment 1}

\section{Participants}

Seventeen healthy adults (10 males, mean age was 22.8 years, age range 19-23 years) from Beijing International Studies University were recruited as participants. All participants were right-handed Chinese native speakers, with normal or corrected-to-normal vision. All participants gave their informed consent prior to the study and got compensation for their participation.

\section{Materials and Procedure}

The experimental stimuli consisted of 90 sets of three sentences. In each set, each sentence contained two clauses. The first clause described an unrealistic fictive event (e.g., Harry Potter was talking with Hermione....), and was identical in all three sentences within the set. The second clause introduced a new character, which could be unrealistic or realistic. Based on the information of the new character in the second clause, the three sentences in each set could be divided into three conditions: (1) Consistent condition, in which the new character was an unrealistic fictional character and fit the event described in the first clause (e.g., the new character and the event belong to the same fictional world) (e.g., Harry Potter was talking with Hermione, and suddenly Lord Voldemort appeared in front of them); (2) Reality Inconsistent condition, in which the new character was a real person and thus its reality was mismatched with the event in the first clause (e.g., Harry Potter was talking with Hermione, and suddenly Bill Gates appeared in front of them); and (3) Fiction Inconsistent 
condition, in which the new character was an unrealistic fictional character, but does not fit the event described in the first clause (e.g., the new character and the event belong to different fictional worlds) (e.g., Harry Potter was talking with Hermione, and suddenly Spider-man appeared in front of them). In each condition, the person's or character's name in the second clause was the critical fragment. To generate the 90 sentences in the Reality Inconsistent condition, 90 real people's names were used in the second clauses of all sentences, including the names of famous artists, athletes, actors, singers, politicians and scientists. One hundred and eighty famous fictive character's names were used in the Fiction Consistent condition and the Fiction Inconsistent condition, including the names of characters from well-known fairy tales, scientific fiction, cartoons, and comics.

Fifteen Chinese native speakers were recruited to rate the experimental stimuli (none of them took part in the ERP experiment). The rating focused on the critical fragment (i.e. the real people's and fictive characters' names in the second clauses). Participants were asked to rate the familiarity of each name using a 7-point scale $(1=$ very unfamiliar, $7=$ very familiar) and the reality of each name (i.e. whether the person/character is a real human being) using a binary scale $(0=n o, 1=y e s)$. Furthermore, participants were instructed to do a cloze test. In this test, they read the first clause of each experimental sentence and then generated the name in the second clause.

Results of one-way ANOVA showed that the critical fragments of the three conditions (i.e. the real people's/characters' names in the second clauses) were significantly different in reality $[F(2,267)=758.60, p<$ 0.001]. Pairwise comparisons with Bonferroni correction showed that the real people's names had higher reality scores than did the fictional characters' names in other two conditions (both $p \mathrm{~s}<0.001$ ). In addition, ANOVA results for cloze probability showed significant differences between three conditions $[F(2,267)=530.06, p<0.001]$. Pairwise comparisons showed that the Consistent condition had higher cloze probability as compared with other two conditions (both $p \mathrm{~s}<0.001$ ). The three conditions were not different in familiarity $[F(2,267)=2.140, p=0.120]$ and word length $[F(2,267)=2.200, p=0.113]($ Table 1$)$.

The 270 experimental sentences were divided into three versions, each of which included 30 sentences for each condition. Each participant only processed one version during the ERP experiment. The three sentences from each set were in three different versions, and thus each participant only read one sentence from each set. To balance the number of the correct sentences and the number of anomalous sentences in the experiment, we added another set of 90 sentences as filler material. In the 90 filler sentences, 60 were semantically correct, and the rest 30 sentences were semantic anomalous. In each semantic anomalous sentence, the final word was incompatible with the sentence meaning. The ordering of the experimental sentences and filler sentences was pseudorandomized. To summarize, during the ERP experiment, each participant processed 180 sentences (i.e. 90 experimental sentences and 90 filler sentences), half of which were correct and half of which were semantically anomalous.

A semantic judgment task was used in the experiment. In this task, participants read each presented sentence carefully and then decided whether the sentence meaning was plausible. Each trial started from a fixation point that was presented on the center of the screen for $300 \mathrm{~ms}$, and then a sentence was presented word by word. Each word was presented $500 \mathrm{~ms}$, followed by a $300 \mathrm{~ms}$ blank interval. A question mark appeared after the final word of the sentence, and it was presented on the screen for $2000 \mathrm{~ms}$. Participants were instructed to give their responses by pressing a button with their left or right index finger. They were asked to make responses as quickly as possible, and as accurate as possible. When a response was given, a new trial started. All participants had a short practice before the experiment to get familiar with the task.

Table 1. Rating results for the experimental stimuli

\begin{tabular}{cccccc}
\hline & Familiarity & Reality & Cloze probability & Length & Relevance \\
\hline Experiment 1 & & & & & \\
Consistent & $5.74(0.66)$ & $0.07(0.25)$ & $0.60(0.28)$ & $2.84(0.72)$ & N/A \\
Fiction Inconsistent & $5.59(0.49)$ & $1.00(0.00)$ & $0.00(0.00)$ & $2.68(0.54)$ & N/A \\
Reality Inconsistent & $5.73(0.51)$ & $0.04(0.21)$ & $0.00(0.00)$ & $2.68(0.58)$ & N/A
\end{tabular}

Experiment 2

$\begin{array}{cccccc}\text { High Relevance } & 4.83(0.87) & 1.00(0.00) & \text { N/A } & 2.67(0.80) & 5.45(0.67) \\ \text { Realistic Irrelevance } & 4.56(0.62) & 1.00(0.00) & \text { N/A } & 2.82(0.72) & 1.03(0.18) \\ \text { Fictional Irrelevance } & 4.70(0.67) & 0.07(0.25) & \text { N/A } & 2.75(0.54) & 1.00(0.00)\end{array}$




\section{Data Acquisition}

Continuous electroencephalogram (EEG) was recorded using 64 active electrodes (ActiCap, Brain Products GmbH, Munich, Germany) according to the standard International 10-20 system. Bilateral mastoids were used as references, and the EEG was grounded to the forehead. Horizontal eye movements were measured by horizontal electrooculogram (HEOG) $\mathrm{Ag} / \mathrm{AgCl}$ electrodes that were placed at the outer canthus of each eye. Vertical eye movements were measured by a vertical electrooculogram (VEOG) recorded from $\mathrm{Ag} / \mathrm{AgCl}$ electrodes placed closely above and below the left eye. During the experiment, all impedances were kept below $5 \mathrm{k} \Omega$, the EEG signals were band-pass filtered between $0.01-100 \mathrm{~Hz}$, and they were amplified and digitized at a rate of $500 \mathrm{~Hz}$ using a BrainAmp amplifier (Brain Products $\mathrm{GmbH})$.

\section{Data Analysis}

The EEGLAB 9.044 (Delorme \& Makeig, 2004) was used to process the collected EEG data. The data were first referenced to the mean of the bilateral mastoids, and then they were filtered with a $0.2 \mathrm{~Hz}$ high-pass filter to remove drift and a $40 \mathrm{~Hz}$ low-pass filter to eliminate line noise. The artifacts caused by eye blinks, eye movements and muscular activity were removed using independent component analysis (ICA) (Onton \& Makeig, 2006). ICA can blindly decompose multichannel EEG data into independent components (ICs) reflecting brain-generated EEG activities or irrelevant artifacts. Each IC has its activity time course and a set of projections to the recording electrodes as its scalp map. In this study, the artifact ICs were removed on the basis of visual inspection. After that, the epoch on the critical fragment (i.e. the new person's/character's name in the second clause of each experimental sentence) was extracted with a time window from $200 \mathrm{~ms}$ before the onset of the fragment to $800 \mathrm{~ms}$ after the fragment onset. In this epoch time window, the effects elicited by fiction inconsistency and reality inconsistency were examined by measuring the mean amplitude of the ERPs for each participant (i.e. the average of non-rejected epochs from 0 to $800 \mathrm{~ms}$ after the fragment onset, which was calculated relative to the baseline from -200 to $0 \mathrm{~ms}$ ).

Two types of effect were examined in Experiment 1, including the N400 effect $(300-450 \mathrm{~ms}$ after the stimulus onset) and the late positivity (500-700 ms after the stimulus onset). For each effect, repeated measures ANOVA was conducted with within-subject factors of Sentence Type (Consistent, Fiction Inconsistent, and Reality Inconsistent) and electrode site from midline sites (4 levels $-\mathrm{Fz}, \mathrm{Cz}, \mathrm{CPz}$, and $\mathrm{Pz}$ ), medial sites (with 2 levels of hemisphere- left and right, and 5 anterior-posterior levels - F1/F2, FC1/FC2, $\mathrm{C} 1 / \mathrm{C} 2, \mathrm{CP} 1 / \mathrm{CP} 2$, and $\mathrm{P} 1 / \mathrm{P} 2$ ), lateral sites (with 2 levels of hemisphere- left and right, and 5 anterior-posterior levels $\mathrm{F} 3 / \mathrm{F} 4, \mathrm{FC} 3 / \mathrm{FC} 4, \mathrm{C} 3 / \mathrm{C} 4, \mathrm{CP} 3 / \mathrm{CP} 4$, and $\mathrm{P} 3 / \mathrm{P} 4)$, and peripheral sites (with 2 levels of hemisphere- left and right, and 5 anterior-posterior levels - F5/F6, FC5/FC6, C5/C6
$\mathrm{CP} 5 / \mathrm{CP} 6$, and P5/P6). For all analyses, we reported the original degrees of freedom. We also applied a Greenhouse-Geisser correction for sphericity to $p$ values when more than two levels of a factor were presented (Greenhouse \& Geisser, 1959). Reliable main effects were followed by pairwise comparisons with Bonferroni correction and reliable interaction effects were followed by simple effects analysis. We reported significant main effects and interactions that involved Sentence Type, since they were of most theoretical interest. Any main effect or interaction effect not reported was insignificant $(p>0.05)$. Marginally significant effects $(0.05<p<0.1)$ were reported only when accompanied by at least one other significant main effect or interaction.

\section{Results}

\section{Behavioral Results}

The semantic judgment results were showed in Table 2. We conducted repeated measures ANOVA with Sentence Type (Consistent, Reality Inconsistent, and Fiction Inconsistent) as a within-subject factor. Results for reaction time showed no significant difference between three conditions $[F(2,32)=1.29, p=0.288]$. Results for accuracy indicated significant Sentence Type effect $[F(2,32)=8.05$, $p=0.002]$. Pairwise comparisons with Bonferroni correction showed that Realistic Inconsistent sentences had higher accuracy than did Consistent sentences $(p=0.007)$ and Fictional Inconsistent sentences $(p=0.016)$. No other effect was found.

Table 2. Behavioral results of Experiment 1 and Experiment 2

\begin{tabular}{ccc}
\hline & Reaction time & Accuracy \\
\hline Experiment 1 & & \\
\hline Consistent & $557.2(112.0)$ & $0.80(0.05)$ \\
\hline Reality Inconsistent & $540.0(106.5)$ & $0.88(0.09)$ \\
\hline Fiction Inconsistent & $571.7(136.4)$ & $0.80(0.10)$ \\
\hline & & \\
\hline Experiment 2 & & \\
\hline High Relevance & $536.0(115.4)$ & $0.77(0.06)$ \\
\hline Realistic Irrelevance & $507.6(124.2)$ & $0.96(0.05)$ \\
\hline Fictional Irrelevance & $582.1(159.0)$ & $0.81(0.12)$ \\
\hline
\end{tabular}

\section{ERP Results}

Figure 1 indicates the ERPs elicited by the critical fragment (real person's name and fictional characters' names) in the second clause. Visual inspection shows that compared with Consistent sentences, Reality Inconsistent sentences and Fiction Inconsistent sentences elicited a stronger N400 effect $(300-450 \mathrm{~ms}$ after stimulus onset) and a late positivity (500-700 ms after stimulus onset).

N400 effect: 300 - $\mathbf{4 5 0}$ ms time window. In the 300-450 ms time window, repeated measure ANOVA for midline sites indicated a main effect for Sentence Type $[F(2,32)=$ $8.23, p=0.002]$, a main effect for Electrode site $[F(3,48)=$ 
6.39, $p=0.003]$, and a significant Sentence Type $\times$ Electrode site interaction $[F(6,96)=4.18, p=0.008]$. Simple effect analyses revealed that Sentence Type effect was significant on $\mathrm{Cz}[F(2,32)=5.52, p=0.009], \mathrm{CPz}[F(2$, $32)=6.47, p=0.004]$, and $\mathrm{Pz}[F(2,32)=7.13, p=0.003]$. Pairwise comparisons with Bonferroni correction indicated that on $\mathrm{Cz}$, Realistic Irrelevance sentences $(p=0.046)$ and Fictional Irrelevance sentences $(p=0.008)$ elicited larger effects than did High Relevance sentences. On CPz, Realistic Irrelevance sentences $(p=0.033)$ and Fictional Irrelevance sentences $(p=0.015)$ elicited larger effects as compared with High Relevance sentences. On Pz, Realistic Irrelevance sentences $(p=0.020)$ and Fictional Irrelevance sentences ( $p$ $=0.003$ ) elicited larger effects than did High Relevance sentences. No other effect was found.

Repeated measure ANOVA for medial sites indicated a main effect for Sentence Type $[F(2,32)=9.28, p=0.002]$, and a significant Sentence Type $\times$ Electrode site interaction $[F(8,128)=8.26, p=0.001]$. Simple effect analyses revealed that Sentence Type effect was significant on $\mathrm{C} 1 / \mathrm{C} 2$ $[F(2,32)=10.78, p<0.001], \mathrm{CP} 1 / \mathrm{CP} 2[F(2,32)=10.98, p$ $<0.001]$, and $\mathrm{P} 1 / \mathrm{P} 2[F(2,32)=7.57, p=0.002]$. Pairwise comparisons with Bonferroni correction indicated that on $\mathrm{C} 1 / \mathrm{C} 2$, Realistic Irrelevance sentences $(p=0.010)$ and Fictional Irrelevance sentences $[p=0.004]$ elicited larger effects than did High Relevance sentences. On CP1/CP2, Realistic Irrelevance sentences $(p=0.019)$ and Fictional Irrelevance sentences $(p=0.003)$ elicited larger effects as compared with High Relevance sentences. On P1/P2, Realistic Irrelevance sentences $(p=0.035)$ and Fictional Irrelevance sentences $(p=0.010)$ elicited larger effects than did High Relevance sentences. No other effect was found.

Repeated measure ANOVA for lateral sites indicated a main effect for Sentence Type $[F(2,32)=7.08, p=0.007]$, and a significant Sentence Type $\times$ Electrode site interaction $[F(8,128)=4.86, p=0.008]$. Simple effect analyses revealed that Sentence Type effect was significant on $\mathrm{C} 3 / \mathrm{C} 4$ $[F(2,32)=8.16, p=0.001], \mathrm{CP} 3 / \mathrm{CP} 4[F(2,32)=7.13, p=$ $0.003]$, and $\mathrm{P} 3 / \mathrm{P} 4[F(2,32)=5.03, p=0.013]$. Pairwise comparisons with Bonferroni correction indicated that on $\mathrm{C} 3 / \mathrm{C} 4$, Realistic Irrelevance sentences $(p=0.017)$ and Fictional Irrelevance sentences $(p=0.001)$ elicited larger effects than did High Relevance sentences. On CP3/CP4, Realistic Irrelevance sentences $(p=0.027)$ and Fictional Irrelevance sentences $(p=0.003)$ elicited larger effects as compared with High Relevance sentences. On P3/P4, Realistic Irrelevance sentences elicited a marginally larger effect than did High Relevance sentences $(p=0.069)$, and Fictional Irrelevance sentences $(p=0.002)$ elicited larger effects than did High Relevance sentences. No other effect was found.

Finally, repeated ANOVA for peripheral sites did not show any significant effect for Sentence Type.

Late positivity: $500-700 \mathrm{~ms}$ time window. In the 500-700 ms time window, repeated measure ANOVA for midline sites indicated a marginal effect for Sentence Type $[F(2,32)=2.75, p=0.099]$, a main effect for Electrode site $[F(3,48)=14.7, p<0.001]$, and a significant Sentence Type $\times$ Electrode site interaction $[F(3,48)=5.17, p=0.004]$. Simple effect analyses revealed that Sentence Type effect was marginally significant on $\mathrm{Cz}[F(2,32)=3.00, p=$ 0.064], and significant on $\mathrm{CPz}[F(2,32)=4.16, p=0.025]$ and $\mathrm{Pz}[F(2,32)=3.79, p=0.033]$. Pairwise comparisons with Bonferroni correction indicated that on $\mathrm{Cz}$, Realistic Irrelevance sentences elicited a marginally larger effect than did High Relevance sentences ( $p=0.063$ ). On CPz, Realistic Irrelevance sentences elicited a larger effect as compared with High Relevance sentences ( $p=0.007)$. On Pz, Realistic Irrelevance sentences elicited a larger effect than did High Relevance sentences $(p=0.006)$. No other effect was found.

Repeated measure ANOVA for medial sites indicated a significant Sentence Type $\times$ Electrode site interaction $[F(8$, $128)=6.12, p=0.001]$. Simple effect analyses revealed that Sentence Type effect was significant on CP1/CP2 $[F(2,32)$ $=4.05, p=0.027]$ and $\mathrm{P} 1 / \mathrm{P} 2[F(2,32)=3.83, p=0.032]$. Pairwise comparisons with Bonferroni correction indicated that on CP1/CP2, Realistic Irrelevance sentences elicited a larger effect as compared with High Relevance sentences ( $p$ $=0.006)$. On P1/P2, Realistic Irrelevance sentences elicited a larger effect than did High Relevance sentences $(p=0.008)$. No other effect was found.

ANOVA lateral sites and peripheral sites revealed no significant effect for Sentence Type. 


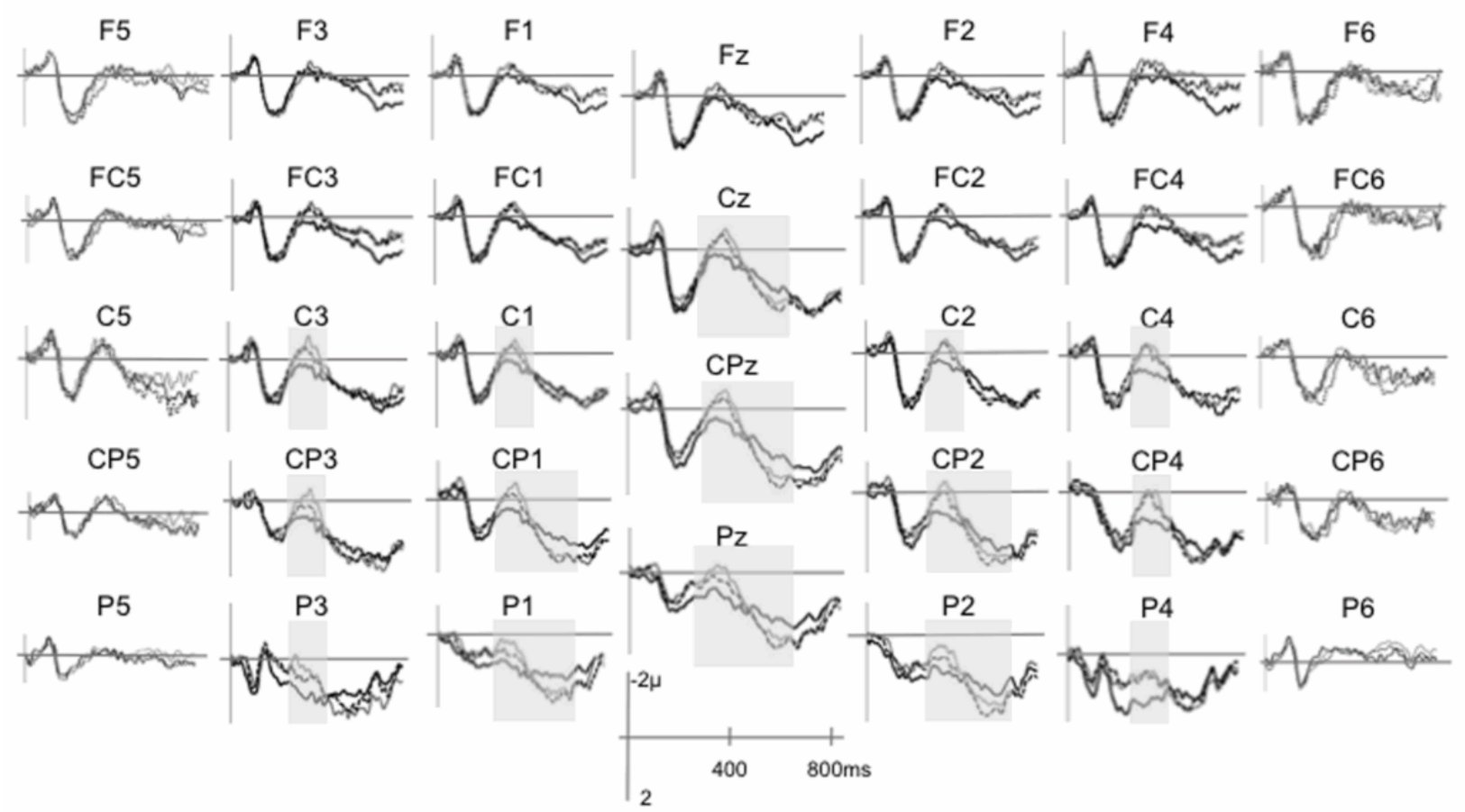

Figure 1. Grand-average ERP results of Experiment 1. The waves in electrodes represent the mean signal evoked by the Consistent sentences (black solid lines), Reality Inconsistent sentences (dashed lines), and Fiction Inconsistent sentences (grey solid lines). Significant effects were marked with light gray color.

\section{Discussion}

The behavioral result indicated higher accuracy in the Realistic Inconsistent sentences than in Fiction Inconsistent sentences and Consistent sentences. This suggests that reality/fiction mismatch is easier to detect relative to fiction/fiction mismatch. The ERP results showed that Fiction Inconsistent sentences elicited a significant N400 effect as compared with Consistent sentences. The Reality Inconsistent sentences also elicited a N400 effect (to a lesser extent) relative to Consistent sentences. Furthermore, in the late time window, the Reality Inconsistent sentences showed a positivity as compared with Consistent sentences.

The different behavioral and ERP results in the Reality Inconsistent sentences and Fiction Inconsistent sentences suggest that reality/fiction distinction and fiction/fiction distinction have different cognitive mechanisms. Fiction/fiction distinction mainly rely on the semantic knowledge about different fictional worlds, and thus it is harder to process Fiction Inconsistent sentences than to process Realistic Inconsistent sentences, and fiction/fiction mismatch only elicited a reliable N400 effect. This N400 effect may reflect difficulty in integrating the new characters with preexisting context. Distinguishing reality from fiction, however, is easier than distinguishing fiction from fiction, and reality/fiction mismatch elicited both an N400 effect and a reliable late positivity. The ERP effects suggest that reality/fiction mismatch not only causes difficulty in semantic integration, but also causes processing difficulty at a later stage or a more global level. These results imply that reality/fiction distinction may not only rely on the semantic knowledge of the real world and fictional worlds.

\section{Experiment 2}

Although Experiment 1 showed that reality/fiction mismatch elicited significant ERP effects, it is still unclear whether reality/fiction distinction merely reflects the distinction between different levels of personal relevance. The aim of Experiment 2 is to examine the reality/fiction distinction after controlling the personal relevance.

\section{Participants}

All participants in Experiment 1 also took part in Experiment 2.

\section{Materials and Procedure}

The stimuli of Experiment included 90 sets of three sentences. Each sentence in each set contained two clauses. In the first clause, the subject was always a second person pronoun (i.e. you). The clause described a common daily-life event (e.g., you are reading a book in the library....), and it was identical in three sentences within each set. The second clause introduced a new character, which could be realistic or unrealistic. The three sentences in each set were divided into three conditions based on the information of the new character in the second clause: (1) High Relevance condition, in which the new character was a real person, and the person has high relevance to the comprehender (e.g., the real person strongly relates to the comprehender's life) (e.g., You are reading a book in the library, suddenly [a person with high relevance] calls your name); (2) Realistic Irrelevance condition, in which the new character was a real person, but the person has low personal relevance to the comprehender 
(e.g., You are reading a book in the library, suddenly Queen Elizabeth II calls your name); and (3) Fictional Irrelevance condition, in which the new character was a fictional character, and it has low personal relevance to the comprehender (e.g., You are reading a book in the library, suddenly Snow White calls your name).

Fifteen participants who did not participate in Experiment 2 were instructed to finish a material generating experiment. The aims of the experiment were to ensure that the first clause of each sentence in each set always described a familiar daily activity. To achieve this goal, each participant was asked to write down 60 daily activities they often do, such as reading books in the library, walking in a park, or having lunch with friends. We selected 45 most common activities reported by all participants. The 45 events were used twice to generate the first clauses of the sentences in the 90 sets of stimuli.

To ensure the second clauses in three conditions introduced new characters with different personal relevance to participants in the ERP experiment, participant who took part in the ERP experiment was asked to provide words about 60 people highly related to his/her life before the experiment. The words could be the names of friends (e.g., John), family members (e.g., mother, father, sister, brother, etc), or other familiar people (e.g., professor Wang). After that, each participant finished a relevance rating task. In this task, each participant rated how frequently the 60 familiar people, 60 famous people, and 60 fictional characters personally interact with him/her in daily-life using a 7-point scale $(1=$ never, $7=$ very frequently $)$. Personal interactions may include living together, doing activities together or having conversations. Participants were also asked to rate the familiarity and reality of the familiar people, famous people, and fictional characters using the same procedure with that in Experiment 1.

Results of one-way ANOVA showed that the words about familiar people, famous people, and fictional characters were significantly different in relevance $[F(2,177)=2417.36, p<$ 0.001]. Pairwise comparisons with Bonferroni correction showed that the familiar people's names had higher relevance scores than did the names in other two conditions (both $p$ s $<0.001$ ). In addition, ANOVA results for reality showed significant differences between three conditions $[F$ $(2,267)=826.00, p<0.001]$. Pairwise comparisons showed that the Fiction Irrelevance condition had lower reality relative to other two conditions (both $p \mathrm{~s}<0.001$ ). The three conditions were not different in the familiarity $[F(2,177)=$ $2.02, p=0.136]$ and word length $[F<1]$ (Table 1).

Based on the rating result, 30 words about people who have strong relevance to each participant's life were selected. These 30 words would be used in the second clauses of the sentences in the High Relevance condition after different versions of material were generated. In addition, 45 famous people's names and 45 fictional characters' names with low relevance were selected based on the rating result. These 90 names were used twice in the second clauses of the sentences in the Realistic Irrelevance condition and the Fictional
Irrelevance condition of the 90 sets of stimuli.

The 270 experimental sentences were divided into three versions, each of which included 30 sentences for each condition. Each participant only processed one version during the ERP experiment. The three sentences from each set were in three different versions, and thus each participant never read two sentences from the same set. For each participant, the second clauses of the 30 High Relevance sentences contained the 30 words about familiar people. Another group of 90 sentences were added as filler materials in each version to balance the number of the correct sentences and the number of anomalous sentences. In the filler sentences, 60 were correct, and the rest 30 sentences contained semantic anomaly in which the final word was incompatible with the sentence meaning. The ordering of the experimental sentences and filler sentences was pseudorandomized.

The task procedure was same with that in Experiment 1.

\section{Data Acquisition and Analysis}

The data collection and analysis were same with that in Experiment 1.

\section{Results}

\section{Behavioral Results}

The semantic judgment results were showed in Table 2. We conducted repeated measures ANOVA with Sentence Type (High Relevance, Realistic Irrelevance, and Fictional Irrelevance) as a within-subject factor. Results for reaction time showed significant difference between three conditions $[F(2,32)=5.10, p=0.013]$. Pairwise comparisons with Bonferroni correction showed that Realistic Irrelevance sentences had shorter reaction time than did Fictional Irrelevance sentences $(p=0.024)$. Results for accuracy indicated significant Sentence Type effect $[F(2,32)=28.53$, $p<.001]$. Pairwise comparisons with Bonferroni correction showed that Realistic Irrelevance sentences had higher accuracy than did High Relevance sentences $(p<0.001)$ and Fictional Irrelevance sentences $(p<0.001)$. No other effect was found.

\section{ERP Results}

The ERPs elicited by the critical word in the second clause were shown in Figure 2. Visual inspection indicates that as compared with High Relevance sentences, Realistic Irrelevance sentences and Fictional Irrelevance sentences elicited slightly larger negativities $(350-450 \mathrm{~ms}$ after stimulus onset) and a larger late positivity (500-700 $\mathrm{ms}$ after stimulus onset).

N400 effect: 350 - 450 ms time window. In the $350-450$ ms time window, repeated measures ANOVA for midline sites, medial sites, lateral sites, and peripheral sites indicated no significant main effect or interaction effect related to Sentence Type (all ps >0.1).

Late positivity effect: 550-700 ms time window. In the 500-700 time window, repeated measure ANOVA for 
midline sites showed a significant main effect for Sentence Type $[F(2,32)=4.14, p=0.029]$, a significant effect for Electrode site $[F(3,48)=15.01, p<0.001]$, and a significant Sentence Type $\times$ Electrode site interaction $[F(6,96)=10.22$, $p<0.001]$. Simple effect analyses revealed that Sentence Type effect was significant on $\mathrm{CPz}[F(2,32)=9.09, p=$ $0.001]$ and $\mathrm{Pz}[F(2,32)=9.47, p=0.001]$. Pairwise comparisons with Bonferroni correction indicated that on $\mathrm{CPz}$, Realistic Irrelevance sentences $(p=0.028)$ and Fictional Irrelevance sentences $(p=0.005)$ elicited larger effects as compared with High Relevance sentences. Similarly, on Pz, Realistic Irrelevance sentences $(p=0.003)$ and Fictional Irrelevance sentences $(p=0.002)$ elicited larger effects than did High Relevance sentences. No other effect was found.

Repeated measures ANOVA for medial sites showed a marginal effect for Sentence Type $[F(2,32)=2.96, p=$ $0.073]$, a significant effect for Electrode site $[F(4,64)=$ 15.50, $p<0.001]$, and a significant Sentence Type $\times$ Electrode site interaction $[F(8,128)=15.43, p<0.001]$. Simple effect analyses revealed that Sentence Type effect was significant on $\mathrm{C} 1 / \mathrm{C} 2$ sites $[F(2,32)=4.38, p=0.021]$, $\mathrm{CP} 1 / \mathrm{CP} 2$ sites $[F(2,32)=9.44, p=0.001]$, and $\mathrm{P} 1 / \mathrm{P} 2$ sites $[F(2,32)=9.40, p=0.001]$. Pairwise comparisons with Bonferroni correction indicated that on $\mathrm{C} 1 / \mathrm{C} 2$ sites, Fictional Irrelevance sentences elicited a marginally larger effect as compared with High Relevance sentences $(p=$ $0.051)$. On CP1/CP2 sites, Realistic Irrelevance sentences ( $p$ $=0.081$ ) elicited a marginally larger effect than did High Relevance sentences, and Fictional Irrelevance sentences elicited larger effect as compared with High Relevance sentences $(p=0.002)$. On P1/P2 sites, Realistic Irrelevance sentences $(p=0.003)$ and Fictional Irrelevance sentences ( $p$ $=0.003$ ) elicited larger effects than did High Relevance sentences. No other effect was found.

Repeated measure ANOVA for lateral sites showed a significant Sentence Type $\times$ Electrode site interaction $[F(8$, $128)=10.53, p<0.001]$ and no other significant effect related to Sentence Type was found. Simple effect analysis revealed that Sentence Type effect was significant on $\mathrm{CP} 3 / \mathrm{CP} 4$ sites $[F(2,32)=5.10, p=0.012]$ and $\mathrm{P} 3 / \mathrm{P} 4$ sites $[F(2,32)=6.76, p=0.004]$. Pairwise comparisons with Bonferroni correction indicated that on $\mathrm{CP} 3 / \mathrm{CP} 4$ sites, Fictional Irrelevance sentences elicited a larger effect as compared with High Relevance sentences ( $p=0.034)$ On P3/P4 sites, Realistic Irrelevance sentences $(p=0.036)$ and Fictional Irrelevance sentences $(p=0.004)$ elicited larger effects than did High Relevance sentences. No other effect was found.

Repeated measure ANOVA for peripheral sites showed a significant Sentence Type $\times$ Electrode site interaction $[F(8$, $128)=4.94, p=0.005]$ and no other significant effect related to Sentence Type was found. Simple effect analyses revealed that Sentence Type effect was significant on $\mathrm{P} 5 / \mathrm{P} 6$ sites $[F(2$, $32)=3.67, p=0.037]$. Pairwise comparisons with Bonferroni correction indicated that Realistic Irrelevance sentences $(p=0.087)$ and Fictional Irrelevance sentences ( $p$ $=0.069)$ elicited marginally larger effects than did High Relevance sentences. No other effect was found.

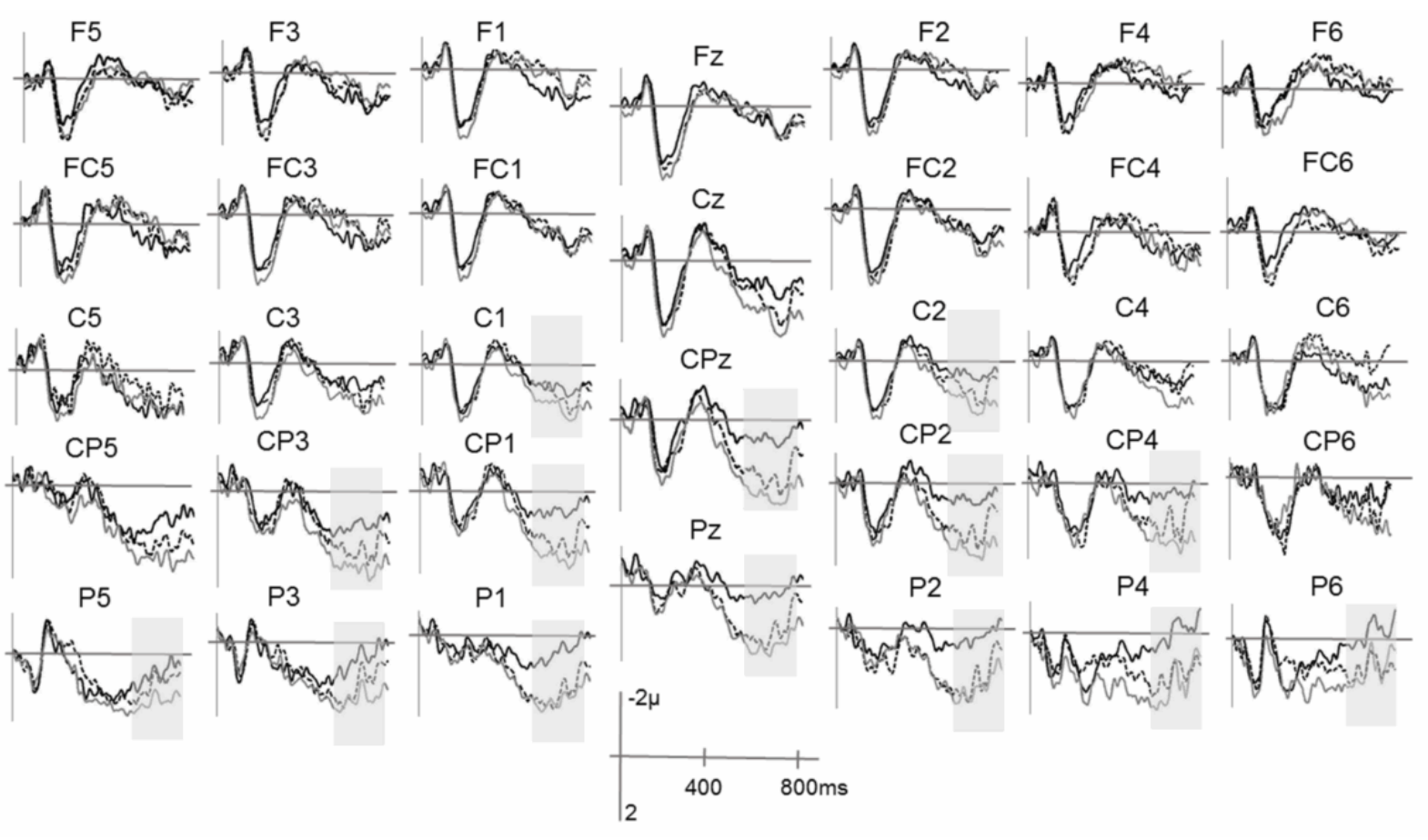

Figure 2. Grand-average ERP results of Experiment 2. The waves in electrodes represent the mean signal evoked by the High Relevance sentences (black solid lines), Realistic Irrelevance sentences (dashed lines), and Fictional Irrelevance sentences (grey solid lines). Significant effects were marked with light gray color. 


\section{Discussion}

The aim of Experiment 2 is to examine the reality/fiction mismatch effect after controlling personal relevance. The prediction is that if reality/fiction distinction is high/low personal relevance distinction, then no extra effect would be elicited by reality/fiction mismatch when the relevance information is controlled.

However, the behavioral results showed that participants had longer reaction time and lower accuracy in the Fiction Irrelevance condition as compared with Realistic Irrelevance condition. This suggests that reality/fiction mismatch became more difficult to be processed as compared with the low relevance condition (i.e. Realistic Irrelevance condition) when participants comprehended events involving themselves. The ERP result indicated that both Irrelevance conditions elicited late positivity as compared with the High Relevance condition, but no significant N400 was found. Furthermore, the late positivity elicited by Realistic Irrelevance sentences was located in the posterior sites, whereas the late positivity induced by Fictional Irrelevance sentences was more extended (i.e. $\mathrm{C} 1 / \mathrm{C} 2$ and $\mathrm{CP} 3 / \mathrm{CP} 4$ ). These results first suggest that reality/fiction distinction is different from the distinction between high and low personal relevance. Second, they suggest that personal relevance and reality/fiction distinction influence sentence comprehension as a late stage.

\section{General Discussion}

Although distinguishing the real world from fictional worlds is a fundamental ability of human beings, the related cognitive mechanism is far from clear. Previous research has investigated the influence of fictional context on the local semantic integration, but it is still unknown how people process the knowledge of reality and fiction, and what factors may influence this processing. The current study used ERPs to address these issues. Experiment 1 investigated reality/fiction distinction and fiction/fiction distinction. Participants read information about a fictional world, and then a real famous person or an imaginative character does not belong to the fictional world was introduced. Results showed that reality/fiction mismatch was easier to be identified as compared with fiction/fiction mismatch. Furthermore, reality/fiction mismatch elicited a N400 effect and a late positivity relative to normal sentences, whereas fiction/fiction mismatch merely elicited an N400 effect. Experiment 2 examined reality/fiction distinction and the distinction between high and low personal relevance. Each participant read information describing a familiar daily activity, and then a real person or a fictional character with low relevance to the participant was introduced into the activity. Results showed that Fictional Irrelevance condition was harder to be processed as compared with Realistic Irrelevance condition. Moreover, Fictional Irrelevance condition elicited a more extended late positivity relative to Realistic Irrelevance condition. Overall, these results suggest that reality/fiction distinction is different from fiction/fiction distinction and the distinction between high and low personal relevance. We will discuss this in more details.

\section{Reality/Fiction Distinction and Fiction/Fiction Distinction}

Reality/fiction distinction has been studied in the cognitive development field for a long time (e.g., Morison \& Gardner, 1978; Samuels \& Taylor, 1994; Sharon \& Woolley, 2004; for a review, see Woolley, 1997). According to Broad (1953), reality/fiction distinction reflects certain basic principles that are commonly accepted as self-evident or established by empirical evidence. Samuels and Taylor (1994) found that children develop this ability quite early in life, and Woolley (1997) suggested that children are not fundamentally different from adults in the ability of distinguishing fiction from reality. Fiction/fiction distinction, on the other hand, has been rarely studied so far (Skolnick \& Bloom, 2006). When people read a novel story or watch a fiction movie, their natural default is to create a unique world for the characters and the world is separate from both the real world and other fictional worlds. One important thing is that creating a fictional world is totally based on the available information about the fictional characters, places, or objects in that world, and the information constitutes the knowledge about the fictional world which is stored in the semantic memory. Thus, reality/fiction distinction involves the use of certain basic principles supported by empirical evidence, whereas fiction/fiction distinction requires the retrieval of semantic knowledge about different fictional worlds. An important difference between the two processes is that reality/fiction distinction can be developed very early in life, and become stable across the life span (Woolley, 1997). The development of fiction/fiction distinction, however, relies on the gain of knowledge about fictional worlds, and can be modified by new information about the fictional worlds (e.g., in movie Shrek, characters from different fictional worlds interact with each other, and this could make the boundaries between fictional worlds flexible).

The behavioral and ERP results in Experiment 1 confirm the difference between reality/fiction distinction and fiction/fiction distinction. Sentences containing reality/fiction mismatch were easy to judge, and they elicited both a N400 effect and a late positivity. This suggests that during sentence comprehension, participants may use some basic principles about the real world to help them decide the semantic plausibility. The use of basic principles influences situation model update. It is possible that during integrating the new character information with preexisting context, a matching between the new character's world and the fictional world described in the first clause is required. If the character is a real person, then the information of the person's world (i.e. the real world) is retrieved, including the basic principles about the real world. These basic principles make the integration between the real person and the fictional world impossible, and may cause a difficulty in constructing the situation model at a global level or structural 
level. The basic assumption here is that our representation of the real world and the representation of fictional worlds are different structurally, and thus reality/fiction mismatch elicits a late positivity. However, this needs to be investigated by future research.

In experiment 1 , sentences containing fiction/fiction mismatch were harder to judge as compared with sentences containing reality/fiction mismatch, and they elicited only a N400 effect. This suggests that fiction/fiction mismatch causes a difficulty in semantic integration, probably because fiction/fiction distinction mainly relies on the semantic knowledge about fictional worlds. Furthermore, since these worlds are all imaginative, anything can happen and no basic principles can be used to distinguish one from another. Thus, for the comprehender, the only way to achieve fiction/fiction distinction may be to retrieve what he/she has read in novels or has watched in movies.

To summarize, reality/fiction distinction may be principle-based and is a fundamental ability of human beings, whereas fiction/fiction distinction is mainly based on the semantic knowledge about fictional worlds.

\section{Reality/Fiction Distinction and High/Low Personal Relevance}

Previous studies have found that reality/fiction distinction and the distinction between high and low personal relevance elicited similar brain activity during language processing. The two types of processes closely relate to each other, because comprehender might have personal interaction with famous people. For example, the comprehender may meet famous people in public, have a chance to talk with them, or take pictures with them. It is possible that the comprehender has episodic memory about famous people and relates these famous people with his/her personal life. However, this is impossible for fictional characters. A comprehender may get to know famous fictional characters only from novels or movies, and there is no way for the comprehender to interact with these fictional characters. Thus, famous people may have higher personal relevance to the comprehender than do fictional characters. Abraham et al. (2008) showed that phrases about famous people elicited stronger activation in the amPFC, precuneus/PCC which are related to episodic memory retrieval and self-referential processing. Abraham and Yves von Cramon (2009) found that entities with higher personal relevance elicited stronger activation in the amPFC and PCC as compared with entities with low personal relevance. These two studies suggest that reality/fiction distinction and high/low personal distinction may share a common neural mechanism.

Nevertheless, the current study indicates that the two types of processes induce different response at behavioral and neural levels. Sentences containing reality/fiction mismatch are more difficult to be judged as "implausible" (e.g., lower accuracy, longer reaction time, and a wider late positivity) relative to low relevance sentences. This is a bit confusing if reality/fiction distinction is a fundamental cognitive ability. One possibility is that the fictional characters in the Fiction
Irrelevance sentences could be interpreted as real people which might have some degree of relevance to participants. For instance, in the sentence "You are watching performance at a concert, suddenly Snow White bows to the audience", Snow White could refer to an actress who was in the costume of Snow White. In the sentence "You are walking on the street, suddenly Decepticons drive a vendor to another street", Decepticons here could metaphorically refer to rude urban management officers. Thus, although these fictional characters have very low personal relevance to participants, they could refer to real people in daily life. This may make the semantic judgment harder than that in the low relevance sentences.

It is important to note that in Experiment 2 both Realistic Irrelevance and Fiction Irrelevance sentences elicited only late positivity, and no reliable N400 effect was found. This suggests that the main influence of low relevance on sentence comprehension is not on the local semantic integration (i.e. relevance processing is not based on semantic knowledge), but on the processes at a global level. Additionally, in Experiment 1, participants comprehended sentences from a third person's perspective, i.e. acting as an observer out of the fictional evens. In Experiment 2, they processed sentences from a second person's perspective, which involved themselves in the described event. In this situation, participants became main characters of the events, and their episodic memory and personal experiences were retrieved during comprehension. The change of perspective and the use of episodic memory might influence the ERP effects. These issues are important and need to be addressed in future research.

\section{Conclusions}

Taken together, the current finding shows that reality/fiction distinction is different from fiction/fiction distinction and high/low personal relevance distinction at behavioral and neural levels. Distinguish reality from fiction is a fundamental ability and may rely on certain basic principles about the real world, whereas distinguishing different fictional worlds is based on semantic knowledge. Reality/fiction mismatch and entities with low relevance can cause processing difficulty at a global level during sentence comprehension, suggesting the importance of the two types of information in building the mental representation of the described situation. Fiction/fiction mismatch, on the other hand, mainly causes difficulty in semantic integration. Future research is needed to clarify what basic principles are critical for reality/fiction distinction, and how these principles constrain the situation model construction and update. Additionally, factors (e.g., perspectives) that may influence reality/fiction during comprehension need to be investigated. 


\section{REFERENCES}

[1] Abraham, A., \& von Cramon, D. Y. (2009). Reality = relevance? Insights from spontaneous modulations of the brain's default network when telling apart reality from fiction. PLoS One, 4, e4741. doi: 10.1371/ journal.pone.0004741

[2] Abraham,A.,vonCramon,D.Y.,\&Schubotz,R.I.(2008).Meetin gGeorge Bush versus meeting Cinderella:The neural response when telling apart what is real from what is fictional in the context of our reality. Journal of Cognitive Neuroscience, 20, 965-976. doi: 10.1162/jocn.2008.20059

[3] Broad, C. D. (1953). Religion, philosophy, and psychical research. New York: Harcourt, Brace.

[4] Cavanna, A. E., \& Trimble, M. R. (2006). The precuneus: A review of its functional anatomy and behavioural correlates. Brain, 129, 564-583.

[5] Delorme, A., \& Makeig, S. (2004). EEGLAB:An open source toolbox for analysis of single-trial EEG dynamics including independent compo- nent analysis. Journal of Neuroscience Methods, 134, 9-21.

[6] Ferguson, H. J., Sanford, A. J., \& Leuthold, H. (2008). Eye-movements and ERPs reveal the time course of processing negation and remitting counterfactual worlds. Brain Research, 1236, 113-125. doi: 10.1016/ j.brainres.2008.07.099

[7] Filik, R., \& Leuthold, H. ( 2008). Processing local pragmatic anomalies in fictional contexts: Evidence from the N400. Psychophysiology, 45, 554-558. doi:

10.1111/j.1469-8986.2008.00656.x

[8] Gilbert, S. J., Dumontheil, I., Simons, J. S., Frith, C. D., \& Burgess, P. W. (2007). Comment on "Wandering minds: the default network and stimulus-independent thought'. Science, 317,43 .

[9] Greenhouse, S. W., \& Geisser, S. (1959). On methods in the analysis of profile data. Psychometrika, 24, 95-112.

[10] Hald, L., Steenbeek-Planting, E., \& Hagoort, P. (2007). The interaction of discourse context and world knowledge in online sentence comprehen- sion. Evidence from the N400. Brain Research, 1146, 210-218. doi: 10.1016/j.brainres.2007.02.054

[11] Kutas, M., \& Federmeier, K. D. (2011). Thirty years and counting: Finding meaning in the N400 component of the event-related brain potential (ERP). Annual Review of Psychology, 62, 621-647. doi: 10.1146/ annurev.psych.093008.131123
[12] Lewis, D. (1978). Truth in fiction. American Philosophical Quarterly, 15, 37-46.

[13] Morison, P., \& Gardener, H. (1978). Dragons and dinosaurs: The child's capacity to differentiate fantasy from reality. Child Development, 49, 642-648.

[14] Nieuwland, M. S. (2013). "If a lion could speak.". Online sensitivity to propositional truth-value of unrealistic counterfactual sentences. Journal of Memory and Language, 68, 54-67. doi: 10.1016/ j.jml.2012.08.003

[15] Nieuwland, M. S., \& Martin, A. E. (2012). If the real world were irrelevant, so to speak: The role of propositional truth-value in counterfactual sentence comprehension. Cognition, 122, 102-109. doi: 10.1016/ j.cognition.2011.09.001

[16] Nieuwland, M. S., \& Van Berkum, J. J. A. (2006). When peanuts fall in love: N400 evidence for the power of discourse. Journal of Cognitive Neuroscience, 18, 1098-1111. doi: 10.1162/jocn.2006.18.7.1098

[17] Onton, J., \& Makeig, S. (2006). Information-based modeling of event- related brain dynamics. Progress in Brain Research, 159, 99-120. doi: 10.1016/S0079-6123(06)59007-7

[18] Ramnani, N., \& Owen, A. M. (2004). Anterior prefrontal cortex: Insights into function from anatomy and neuroimaging. Nature Reviews Neuroscience, 5, 184-194

[19] Samuels, A., \& Taylor, M. (1994). Children's ability to distinguish fantasy events and real-life events. British Journal of Developmental Psychology, 12, 417-427.

[20] Skolnick, D., \& Bloom, P. (2006). What does Batman think about SpongeBob? Children's understanding of the fantasy/fantasy distinction. Cognition, 101, B9-B18. doi: 10.1016/j.cognition.2005.10.001

[21] Sharon, T., \& Woolley, J. D. (2004). Do monsters dream? Young children's understanding of the fantasy/reality distinction. British Journal of Developmental Psychology, 22, 293-310.

[22] Woolley, J. D. (1997). Thinking about fantasy: are children fundamentally different thinkers and believers from adults? Child Development, 68, 991-1011.

[23] Yang, J., \& Xue, J. (2014). Distinguishing different fictional worlds during sentence comprehension: ERP evidence. Psychophysiology. 51, 42-51. doi: 10.1111/psyp.12149. Epub 2013 Oct 22.

[24] Zwaan, R. A., \& Radvansky, G. A. (1998). Situation models in language comprehension and memory. Psychological Bulletin, 123, 162-185. 